\title{
A Triad of Unilateral Renal Dysgenesis with Ipsilateral Seminal Vesical and Ejaculatory Duct Obstruction: An Uncommon Urogenital Congenital Anomaly, Zinner Syndrome-A Case Report
}

\author{
Rajani Gorantla ${ }^{1}$ Sameera Allu ${ }^{1}$ Ankamma Rao ${ }^{1}$ \\ ${ }^{1}$ Department of Radiodiagnosis, NRI Medical College and Hospital, \\ Mangalagiri, Andhra Pradesh, India \\ Indian J Radiol Imaging 2021;31:707-709. \\ Address for correspondence Sameera Allu, MD, DNB, King George \\ Hospital, Visakhapatnam, Andhra Pradesh 530017, India \\ (e-mail: allu.sameera90@gmail.com).
}

\begin{abstract}
Zinner syndrome is a rare congenital anomaly of the urogenital system resulting from an in utero insult during the first trimester. This entity comprises a triad of unilateral renal agenesis/dysgenesis with ipsilateral seminal vesical and ejaculatory duct obstruction. This combination of urinary and genital abnormalities occurs because of the closely related embryological origin of these structures from the distal mesonephric

Keywords

- Zinner syndrome

- urogenital

- mesonephric duct (Wolffian) duct. Nearly 200 cases of seminal vesical cysts with ipsilateral renal agenesis have been reported in the literature. The affected person generally presents in early adulthood when the reproductive activity commences. In this report, we present a case of a 22-year-old male with complaints of painful ejaculation.
\end{abstract}

\section{Introduction}

Zinner syndrome (ZS) consists of a triad of unilateral renal agenesis/dysgenesis, ipsilateral seminal vesical cysts, and ipsilateral ejaculatory duct obstruction resulting from the developmental arrest of the caudal mesonephric duct. This entity is one of the rarest congenital anomalies, with the frequency reported to be $0.0046 \%{ }^{1}$ Two hundred cases of seminal vesicle cysts associated with ipsilateral renal agenesis have been reported in the literature until 2009. ${ }^{2}$ The diagnosis of this entity is primarily made with the help of imaging. Magnetic resonance imaging (MRI) helps to rule out other causes of pelvic cysts with clear delineation of the anatomical structures.

\section{Case History}

A 22-year-old male patient presented to the internal medicine outpatient department with complaints of painful ejaculation and occasional backache more on the right side. There were no other complaints of lower urinary tract symptoms, trauma, or hematospermia. Past medical and surgical history was insignificant. Physical examination was unremarkable. Semen analysis revealed oligospermia with a sperm concentration of $8.5 \mathrm{million} / \mathrm{mL}$. The radiological investigation was initiated with an ultrasound examination of the abdomen and scrotum. A large lobulated tubular cystic lesion was noted in the pelvis in retro vesical space indenting the right posterolateral aspect of the urinary bladder, measuring approximately $6 \times 3 \mathrm{~cm}$. There was no evidence of communication with the urinary bladder. The lesion was anechoic with no internal echoes or soft tissue components. The right seminal vesicle was not visualized. The left seminal vesicle appeared normal (- Fig. 1a, b). On the sonological examination of the upper abdomen, the right kidney was not visualized in the right renal fossa. It could not be traced elsewhere in the abdomen due to poor window from bowel gas. The left kidney was enlarged with preserved
DOI https://doi.org/ 10.1055/s-0041-1735503. ISSN 0971-3026.

\footnotetext{
(C) 2021. Indian Radiological Association. All rights reserved. This is an open access article published by Thieme under the terms of the Creative Commons Attribution-NonDerivative-NonCommercial-License, permitting copying and reproduction so long as the original work is given appropriate credit. Contents may not be used for commercial purposes, or adapted, remixed, transformed or built upon. (https://creativecommons.org/ licenses/by-nc-nd/4.0/)

Thieme Medical and Scientific Publishers Pvt. Ltd., A-12, 2nd Floor, Sector 2, Noida-201301 UP, India
} 

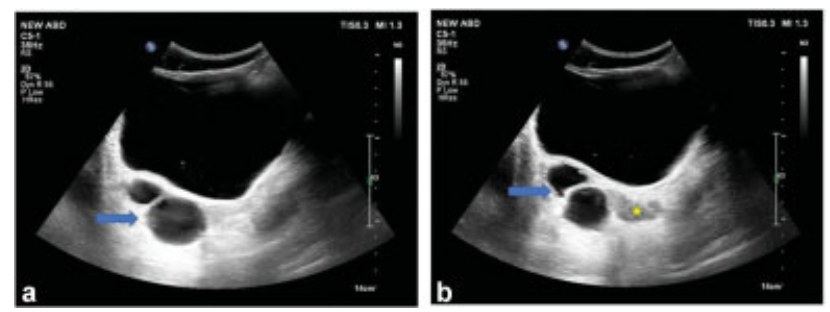

Fig. 1 ( $\mathbf{a}$ and $\mathbf{b}$ ) Ultrasound images of the pelvis in transverse plane at the level of urinary bladder, showing cystic lesion in retrovesical region (blue arrow) on right side with nonvisualization of right seminal vesicle. The smaller cystic lesion of the lateral aspect could represent the dilated ureter as visualized in the MRI. Left seminal vesicle is visualized and appears normal (yellow star).

corticomedullary differentiation in its orthotopic position (-Fig. 2a, b). Bilateral testicles were normal with mildly dilated right vas deferens. Mild hydrocoele was noted in the right scrotal sac. The rest of the study was essentially normal.

After that, the patient was submitted to the MRI for further evaluation of the abnormal findings. The sonological findings were confirmed on MRI. The retro vesical cystic lesion was diagnosed as the right seminal vesical cyst, deciphered from precise anatomical detail from MRI (-Fig. 3). There was an elongated, dilated, tubular cystic lesion seen in the right paramedian region in the pelvis, extending superiorly from the seminal vesical cyst with a blind end at the level of L5-S1 vertebra. This structure was showing fluid signal intensity with $\mathrm{T} 1$ hypointense and $\mathrm{T} 2$ hyperintense signal. There was no evidence of any renal tissue noted at its proximal end (-Figs. 4 and 5). The right kidney was not visualized anywhere in the abdomen. This structure was considered a dilated remnant right ureter with ectopic drainage into the right seminal vesicle based on the anatomical location and morphology ( - Figs. 6 and $\mathbf{4 a}$ ). The left kidney was noted in the left renal fossa with compensatory hypertrophy ( $\mathbf{- F i g .} \mathbf{4 b}$ ). These imaging features of a seminal vesical cyst, ipsilateral renal agenesis with rudimentary ureteric bud, and mild ectasia of vas deferens (probably due to obstruction) were consistent with the diagnosis of ZS, portraying the classic triad. The patient was referred to the Urology department for cyst aspiration, which was successfully performed. The patient did not show up for the followup visits.
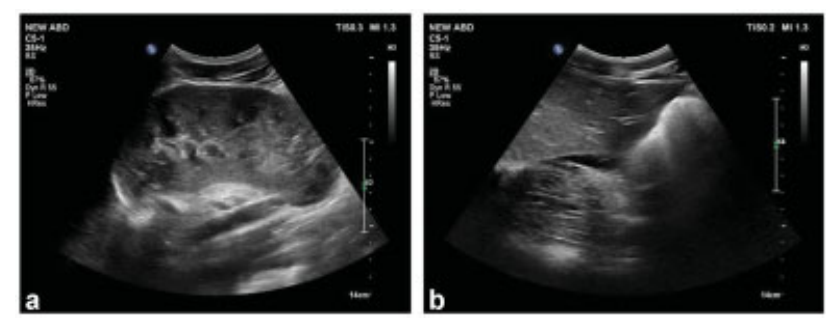

Fig. 2 ( $\mathbf{a}$ and $\mathbf{b}$ ) Ultrasound images showing left kidney appears enlarged with preserved corticomedullary differentiation in the left renal fossa due to compensatory hypertrophy (a). Right kidney is not visualized in the right renal fossa (b).

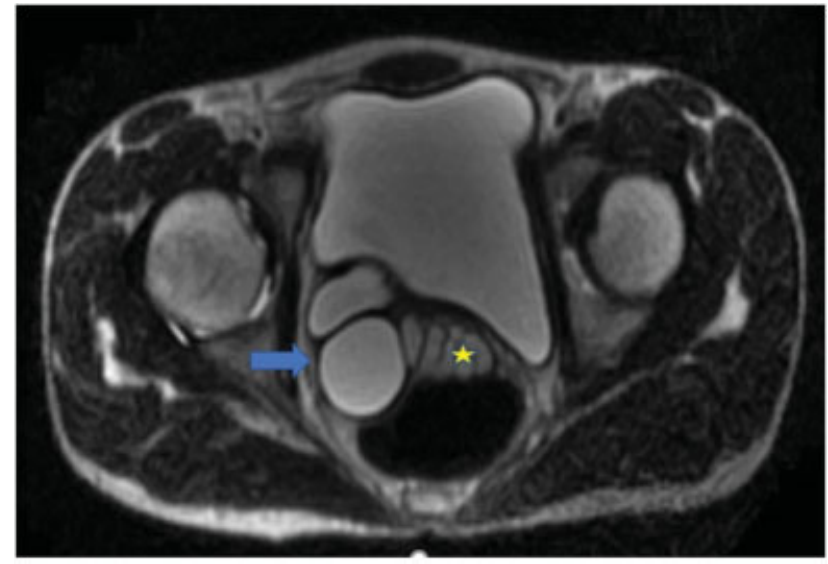

Fig. 3 Axial T2 SSFSE MRI image at the level of pelvis showing retrovesical lobulated cystic lesion on the left side (blue arrow) indenting the posterolateral aspect of the adjacent urinary bladder. Left seminal vesical appears normal (yellow star).
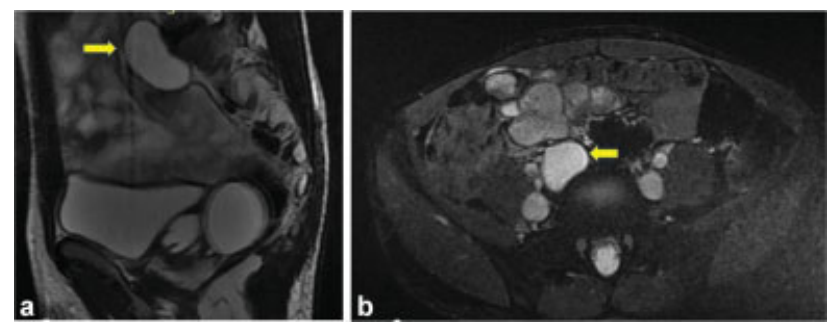

Fig. 4 Sagittal frFSE T2 (a) and axial 2D FIESTA fat sat (b) MR images showing dilated remnant right ureter with proximal blind ending (yellow arrows).
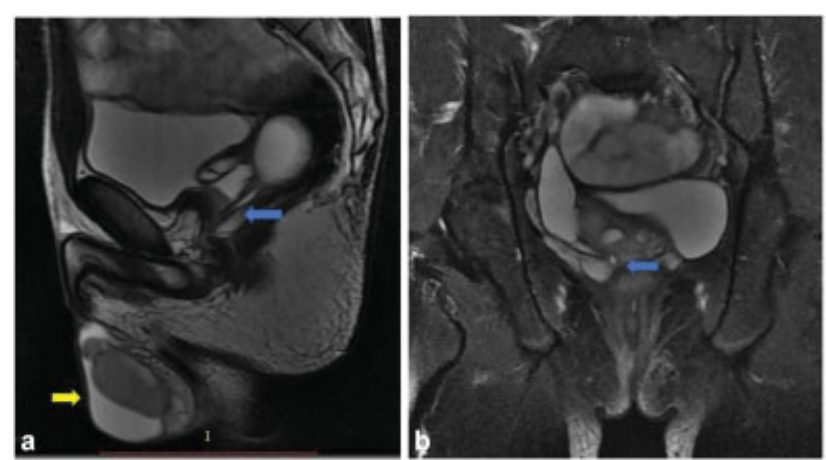

Fig. 5 Sagittal frFSE T2 (a) and coronal frFSE T2 fat sat (b) MRI images of pelvis showing ectopic insertion of right dilated remnant ureter into ipsilateral seminal vesical (blue arrow) and right mild hydrocoele (yellow arrow).

\section{Discussion}

$\mathrm{ZS}$ is one the rarest urogenital congenital anomaly, comprising of a triad of seminal vesical cysts, ipsilateral renal agenesis, and ejaculatory duct obstruction. This entity was first identified by Zinner in 1914, ${ }^{3}$ and after that, 200 cases of seminal vesical cysts with ipsilateral renal agenesis were reported in the literature until 2009. ${ }^{2}$ The patients usually 


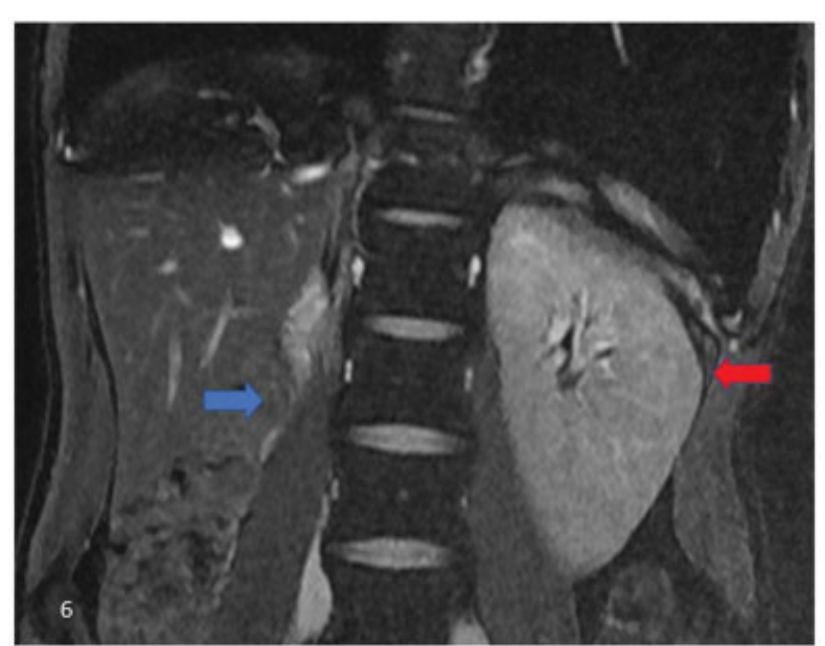

Fig. 6 Coronal 2D FIESTA fatsat MRI image of the upper abdomen showing absence of right kidney in right renal fossa (blue arrow) and compensatory hypertrophy of left kidney in left renal fossa (red arrow).

present in their second and third decades when the reproductive activity is initiated, and symptoms include lower urinary tract symptoms, painful ejaculation, hematospermia, and infertility. ${ }^{4}$ The present case is a 22 -year-old male patient with symptoms of painful ejaculation.

This combination of urinary and genital abnormalities occurs in ZS because of their common embryonic origin from the mesonephric duct. The ureteral bud arises as a diverticulum from the distal mesonephric duct and grows cranially to come in contact with the metanephric blastema and induce its differentiation into the definitive adult kidney. An intrauterine insult occurring in the first trimester can cause abnormal development of the distal mesonephric duct. This maldevelopment results in atresia of the ejaculatory duct with retrograde cystic dilatation of seminal vesicles and abnormal ureteral bud development leading to renal agenesis/dysgenesis.

The main diagnostic challenge lies in the accurate evaluation of cystic lesions in the male pelvis. Different imaging modalities complement each other to arrive at a definitive diagnosis of these cysts with excellent anatomical detailing of the organ of origin. The differential diagnosis of these pelvic cysts includes Wolffian duct derivatives (seminal vesical cysts and ejaculatory duct cysts), Mullerian duct derivatives (prostatic utricular cysts and Mullerian duct cysts), hydronephrotic pelvic kidney or ureter, bladder diverticula, and ureterocoeles. ${ }^{2,5,6}$ They are distinguished depending on their relative position with the bladder neck, communication with the urethra, and associated urogenital anomalies. $^{6,7}$ Ultrasound is the first line of investigation to evaluate these lesions, ${ }^{6}$ which usually raises an alarm for further investigation by MRI or CT to arrive at a final diagnosis. ${ }^{8}$ CT provides better visualization of the associated renal anomalies and the pelvic anatomical structures. Nevertheless, MRI accurately depicts the relationship and communication of the cystic lesion with the adjacent structures. $^{9}$
The management includes surveillance of asymptomatic patients with transrectal sonography preferably. Symptomatic cases are treated with surgical aspiration of the seminal vesicle cysts through perineal or laparoscopic approach or percutaneous cyst drainage or a transurethral cyst deroofing to relieve the ejaculatory duct obstruction. ${ }^{10}$

Patients of ZS, a rare urogenital congenital anomaly, present with nonspecific symptoms of the lower urinary tract, and the diagnosis primarily depends on imaging studies. Two-thirds of congenital seminal vesical cysts are associated with abnormal development of other ipsilateral mesonephric duct derivatives of the urinary and genital system. ${ }^{2,6}$

Whenever seminal vesical cysts are encountered it is always better to check for associated urogenital anomalies, suspecting ZS which can be diagnosed with the triad of seminal vesical cysts, ipsilateral renal agenesis, and ejaculatory duct obstruction, of which the former two components of the triad can be diagnosed with various imaging modalities which are complementary to each other and help provide an accurate diagnosis, among which MRI is considered to be the prime modality of choice for imaging the genital tract developmental anomalies.

\section{Funding}

None.

Conflict of Interest

None.

\section{References}

1 Farooqui A, AlDhahir L, Mahfooz AB. Massive seminal vesicle cyst with ipsilateral renal agenesis-Zinner syndrome in a Saudi patient. Urol Ann 2018;10(03):333-335

2 Pereira BJ, Sousa L, Azinhais P, et al. Zinner's syndrome: an up-todate review of the literature based on a clinical case. Andrologia 2009;41(05):322-330

3 Zinner A. Ein fall von intravesikaler samenblasenzyste. Wien Med Wochenschr 1914;64:604-609

4 Ghonge NP, Aggarwal B, Sahu AK. Zinner syndrome: a unique triad of mesonephric duct abnormalities as an unusual cause of urinary symptoms in late adolescence. Indian J Urol 2010;26(03): 444-447

5 Sheih CP, Hung CS, Wei CF, Lin CY. Cystic dilatations within the pelvis in patients with ipsilateral renal agenesis or dysplasia. J Urol 1990;144(2 Pt 1):324-327

6 Hong YK, Onal B, Diamond DA, Retik AB, Cendron M, Nguyen HT. Robot-assisted laparoscopic excision of symptomatic retrovesical cysts in boys and young adults. J Urol 2011;186(06):2372-2378

7 Dahms SE, Hohenfellner M, Linn JF, Eggersmann C, Haupt G, Thüroff JW. Retrovesical mass in men: pitfalls of differential diagnosis. J Urol 1999;161(04):1244-1248

8 Kanavaki A, Vidal I, Merlini L, Hanquinet S. Congenital seminal vesicle cyst and ipsilateral renal agenesis (Zinner Syndrome): a rare association and its evolution from early childhood to adolescence. European J Pediatr Surg Rep 2015;3(02):98-102

9 Arora SS, Breiman RS, Webb EM, Westphalen AC, Yeh BM, Coakley FV. CT and MRI of congenital anomalies of the seminal vesicles. AJR Am J Roentgenol 2007;189(01):130-135

10 van den Ouden D, Blom JHM, Bangma C, de Spiegeleer AHV. Diagnosis and management of seminal vesicle cysts associated with ipsilateral renal agenesis: a pooled analysis of 52 cases. Eur Urol 1998;33(05):433-440 